\title{
A numerical model for the $\gamma$-ray emission of the microquasar LS 5039
}

\author{
V. Bosch-Ramon and J. M. Paredes
}

\author{
Departament d'Astronomia i Meteorologia, Universitat de Barcelona, Av. Diagonal 647, 08028 Barcelona, Spain \\ e-mail: vbosch@am.ub.es, jmparedes@ub.edu \\ Received 3 November 2003 / Accepted 6 January 2004
}

\begin{abstract}
The possible association between the microquasar LS 5039 and the EGRET source 3EG J1824-1514 suggests that microquasars could also be sources of high-energy $\gamma$-rays. In this paper, we explore, with a detailed numerical model, if this system can produce the emission detected by EGRET $(>100 \mathrm{MeV})$ through inverse Compton (IC) scattering. Our numerical approach considers a population of relativistic electrons entrained in a cylindrical inhomogeneous jet, interacting with both the radiation and the magnetic fields, taking into account the Thomson and Klein-Nishina regimes of interaction. The computed spectrum reproduces the observed spectral characteristics at very high energy.
\end{abstract}

Key words. X-rays: binaries - stars: individual: LS 5039 - gamma-rays: observations - gamma-rays: theory

\section{Introduction}

Microquasars are a selected class of X-ray binaries that produce relativistic radio jets (Mirabel \& Rodríguez 1999; Fender 2004). The origin of the jets is related to the matter accreted by the compact object, a neutron star or a black hole, from the companion star. These systems behave as scaled-down versions of quasars and active galactic nuclei. The population of microquasars is still a very reduced one, with about sixteen known objects up to now (Ribó 2002). Several models have been developed to explore the high energy emission from the jets of microquasars. Two kinds of model can be found in the literature depending on whether hadronic or leptonic jet matter dominates the emission at such an energy range: the hadronic jet models (e.g. Romero et al. 2003), and the leptonic jet models. Among leptonic jet models, there are IC jet emission models that can produce X-rays and $\gamma$-rays, based in some cases on the synchrotron self-Compton (SSC) process (i.e. Band \& Grindlay 1986; Atoyan \& Aharonian 1999), and in other cases on external sources for the IC seed photons (EC) (i.e. Kaufman Bernadó et al. 2002; Georganopoulos et al. 2002). In addition, there are synchrotron jet emission models that can produce X-rays (i.e. Markoff et al. 2001).

The microquasar nature of LS 5039 was clearly established when non-thermal radiation produced in a jet was detected (Paredes et al. 2000). Interest in this source has grown significantly because it has turned out to be a source of persistent radio emitting relativistic jets (Paredes et al. 2002), and because of its proposed association with the unidentified source

Send offprint requests to: V. Bosch-Ramon,

e-mail: vbosch@am.ub.es
3EG J1824-1514 (Paredes et al. 2000) of the 3rd EGRET catalogue (Hartman et al. 1999). Recently, Collmar (2003) reported the detection of an unidentified $\gamma$-ray source by the COMPTEL experiment, and LS 5039 is one of the possible counterparts. We are interested in investigating if microquasars are able to generate the high-energy $\gamma$-ray emission detected by EGRET ${ }^{1}$, using a numerical model based on IC scattering, taking into account energy losses, EC and SSC interactions. In this paper, we focus our attention on the microquasar LS 5039. In Sect. 2 we present the physical scenario of our model, and the model itself is described in Sect. 3. We apply the model to the microquasar LS 5039 in Sect. 4, and the obtained results are discussed in Sect. 5.

\section{The physical scenario}

The scenario of our model consists on an X-ray binary system where the compact object has a disk coplanar with the orbital plane and a jet not necessarily perpendicular to it. It includes also a hot spherical region, called corona, surrounding the compact object. We assume that the leptons dominate the radiative processes that generate $\gamma$-rays. Relativistic electrons, already accelerated and flowing away into the jet, are exposed to photons from the star (assumed to be an isotropic photon field) as well as to photons from the synchrotron emission of the same population of electrons, since we suppose the presence of a magnetic field. This magnetic field is tangled in such a way that the resulted synchrotron emission is isotropic in the jet's reference frame. Although some features like disk-jet interaction (e.g. for blazars, Dermer et al. 1992), reacceleration

\footnotetext{
${ }^{1}$ http://cossc.gsfc.nasa.gov/egret
} 
of the jet's particles (e.g. Markoff et al. 2001), corona-jet interaction and pair creation-annihilation phenomena (e.g. both for Cygnus X-1, Romero et al. 2002) could be significant at some level, their study is beyond the scope of this paper and they will be treated in upcoming works. The $\gamma$-ray emitting region, the $\gamma$-jet, is assumed to be closer to the compact object than the observed radio jets.

\section{Description of the model}

We have developed a numerical IC model for the $\gamma$-ray emission from relativistic electrons in a cylindrical jet (the $\gamma$-jet). This $\gamma$-jet is assumed to be short enough to allow the magnetic field $\left(B_{\gamma}\right)$ to be considered constant. We have included the interaction between the relativistic electrons and both the magnetic and the radiation fields. The energy losses of the leptonic plasma in the $\gamma$-jet are due to its own synchrotron emission, the SSC scattering and the EC scattering of the stellar photons by the relativistic electrons. Due to the strong losses, the electron energy distribution density along the $\gamma$-jet model varies significantly, and in this sense the $\gamma$-jet is non-homogeneous. Thus, the $\gamma$-jet is studied by splitting it into cylindrical transverse cuts or slices. The size of the slices has to be suitable to get almost homogeneous physical conditions within them (energy densities for the radiation and the electrons).

The code used for our $\gamma$-jet model is run in two steps. The first computes, for each slice, both the total radiation energy densities and the radiation energy densities per frequency unit from both the star and the synchrotron process. Then, the calculated radiation energy densities are introduced jointly with the electron distribution function (see Sect. 3.1) in the second step of the program to calculate the IC emission at each slice. Because such slices evolve due to the changes of both the electron energy and the radiation energy densities, the resulting IC radiation will be different depending on the age of the slice (i.e. depending on the distance to the compact object). Once we have computed the IC emission from all the slices, we can sum the contribution of all of them to get the whole emission. Since our model is applied to a wide range of energies for both the electrons and the seed photons, we have taken into account both the low and the high energy regimes (i.e. the Thomson and Klein-Nishina regimes). We have used the cross section of Blumenthal \& Gould (1970). The free parameters of the model are just three: $B_{\gamma}$, the maximum electron Lorentz factor $\left(\gamma_{\mathrm{e} 0}^{\max }\right)$ and the leptonic kinetic luminosity or leptonic jet power $\left(L_{\mathrm{ke}}\right)$.

\subsection{Energy distribution of the electrons}

The differential equation that describes the electron energy evolution in our model is:

$\frac{\mathrm{d} E}{\mathrm{~d} t}=-\left(a_{\mathrm{s}} B_{\gamma}^{2}+a_{\mathrm{c}} U_{\mathrm{syn}}+a_{\mathrm{c}} U_{\text {star }}\right) E^{2}$

where $a_{\mathrm{s}}$ and $a_{\mathrm{c}}$ are constants (of values $2.37 \times 10^{-3}$ and $3.97 \times 10^{-2}$ respectively, in cgs units), $U_{\text {star }}$ is the companion star's radiation energy density, $U_{\text {syn }}$ is the synchrotron radiation energy density, computed using the local approximation (Ghisellini et al. 1985), and $E$ is the electron energy. It is worth noting that we work now in the jet's reference frame. Solving this equation for the given conditions, after some algebra we get the electron Lorentz factor evolution function:

$$
\begin{aligned}
& \gamma_{\mathrm{e}}=\gamma_{\mathrm{e} 0}\left(1+\gamma_{\mathrm{e} 0} m_{\mathrm{e}} c^{2}\left[C_{\mathrm{s}}\left(t-t_{0}\right)\right.\right. \\
& \left.\left.+\frac{C_{\mathrm{c}}}{v_{\mathrm{jet}}^{2} t^{\prime} \sin \varpi}\left(\arctan \frac{t-t^{\prime} \cos \varpi}{t^{\prime} \sin \varpi}-\arctan \frac{t_{0}-t^{\prime} \cos \varpi}{t^{\prime} \sin \varpi}\right)\right]\right)^{-1}
\end{aligned}
$$

where $t$ is the time and $t_{0}$ is the starting time in slice evolution. Electrons are injected at a distance $z_{0}$ to the compact object, which corresponds to the time $t_{0}$, and their evolution continues along the jet axis (to farther $z$ ). $v_{\text {jet }}$ is the jet velocity, $\varpi$ is the angle between the jet and the orbital plane, and $\gamma_{\mathrm{e} 0}$ is the value for the electron Lorentz factor at $t_{0} . R_{\text {orb }}$ is the orbital radius of the binary system, and $t^{\prime}$ is $R_{\text {orb }} / v_{\text {jet }} . C_{\mathrm{s}}$ is $\left(a_{\mathrm{s}} B_{\gamma}^{2}+a_{\mathrm{c}} U_{\mathrm{syn}}\right)$, and $C_{\mathrm{c}}$ is $a_{\mathrm{c}} \Gamma_{\text {jet }} L_{\mathrm{star}} / 4 \pi c$, where $L_{\mathrm{star}}$ is the bolometric luminosity of the companion star, $\Gamma_{\text {jet }}$ is the Lorentz factor of the jet and $c$ is the speed of light. We describe the energy distribution of the (injected) electrons, $N$, with a power law in the slice at $t_{0}$. The initial slice (like the following ones) is assumed to be spatially homogeneous and the particle motion direction follows an isotropic distribution (in the reference frame of the jet). So, at $t_{0}$ :

$N\left(\gamma_{\mathrm{e} 0}\right)=Q \gamma_{\mathrm{e} 0}^{-p}$, for $\gamma_{\mathrm{e} 0}^{\min } \leq \gamma_{\mathrm{e} 0} \leq \gamma_{\mathrm{e} 0}^{\max }$

where $Q$ is the normalization constant of the electrons, related to the jet power (Georganopoulos et al. 2002), and $p$ is the power law index of the electron energy distribution function. $\gamma_{\mathrm{e} 0}^{\max }$ is the maximum Lorentz factor of the electrons in the first slice, which will be treated later. $\gamma_{\mathrm{e} 0}^{\mathrm{min}}$ is the minimum electron Lorentz factor in the first slice, whose value is determined according to both the upper limit of the seed photon energy and the lower limit of the outgoing photon energy (see Sect. 3.3). Taking into account the conservation of the number of particles, $N\left(\gamma_{\mathrm{e}}, t\right) \mathrm{d} \gamma_{\mathrm{e}}=N\left(\gamma_{\mathrm{e} 0}\right) \mathrm{d} \gamma_{\mathrm{e} 0}$, the time evolution of Eq. (3) is found:

$$
\begin{aligned}
N\left(\gamma_{\mathrm{e}}, t\right)= & Q \gamma_{\mathrm{e}}^{-p}\left[1-\gamma_{\mathrm{e}} m_{\mathrm{e}} c^{2}\left(C_{\mathrm{s}}\left(t-t_{0}\right)+\frac{C_{\mathrm{c}}}{v_{\mathrm{jet}}^{2} t^{\prime} \sin \varpi}\right.\right. \\
& \left.\left.\times\left(\arctan \frac{t-t^{\prime} \cos \varpi}{t^{\prime} \sin \varpi}-\arctan \frac{t_{0}-t^{\prime} \cos \varpi}{t^{\prime} \sin \varpi}\right)\right)\right]^{p-2}
\end{aligned}
$$

\subsection{The radiation fields}

Assuming that the stellar photon energy density is isotropic, $U_{\text {star }}$ in the reference frame of the jet is given by $\Gamma_{\text {jet }} L_{\mathrm{star}} / 4 \pi c d^{2}$, where $d$ is the distance between the companion star and a certain slice of the $\gamma$-jet. Assuming that the star emits like a black-body and taking into account its spectral type (see Sect. 4.1), we can obtain the radiation energy density per frequency unit $\left(U_{v \text { star }}\right)$. The numerical calculation of $U_{\text {syn }}$ is difficult when this seed photon field becomes the dominant one. The problem is to know previously the evolution of the $U_{\text {syn }}$ within a slice along the $\gamma$-jet. To know $U_{\text {syn }}, N\left(\gamma_{\mathrm{e}}, t\right)$ has to be 
determined, but $N\left(\gamma_{\mathrm{e}}, t\right)$ depends on the value of $U_{\text {syn }}$ in the previous slice, as can be seen in Eq. (4). We solve this in the following way: $U_{\text {syn }}$ and the radiation energy density per frequency unit $\left(U_{v \text { syn }}\right)$ are found initially starting with Eq. (3), determining their own values for the next step and so on. The synchrotron emission is numerically integrated following the standard formulae from Pacholczyk (1970). The two integrals for computing numerically the emission and absorption coefficients are:

$j_{v}(z)=c_{3} B_{\gamma} \int_{\gamma_{\mathrm{e}}^{\min }(z)}^{\gamma_{\mathrm{e}}^{\max }(z)} N\left(\gamma_{\mathrm{e}}, z\right) F\left(v / v_{\mathrm{c}}\right) \mathrm{d} \gamma_{\mathrm{e}}$

$k_{\nu}(z)=-\frac{c_{3} B_{\gamma} c^{2}}{2 m_{\mathrm{e}} c^{2} v^{2}} \int_{\gamma_{\mathrm{e}}^{\min }(z)}^{\gamma_{\mathrm{e}}^{\max }(z)} \frac{\mathrm{d}}{\mathrm{d} \gamma_{\mathrm{e}}}\left(\frac{N\left(\gamma_{\mathrm{e}}, z\right)}{\gamma_{\mathrm{e}}^{2}}\right) \gamma_{\mathrm{e}}(z)^{2} F\left(v / \nu_{\mathrm{c}}\right) \mathrm{d} \gamma_{\mathrm{e}}(6)$

where $c_{3}=1.87 \times 10^{-23}$ (in cgs units) and $F\left(v / v_{\mathrm{c}}\right)=$ $x \int_{x}^{\infty} k_{5 / 3}(z) \mathrm{d} z$, in which $K_{5 / 3}(z)$ is the Bessel function of the second kind. $v$ and $v_{\mathrm{c}}$ are the frequency and the characteristic frequency respectively. After some algebra, $U_{v \text { syn }}$ is obtained.

\subsection{IC interaction}

The code developed for reproducing the radiation-matter IC interaction operates over files created by the first step. The seed photon energies $\left(\epsilon_{0}\right)$ should be such that the scattered photons had energies inside the chosen energy band following the relationship:

$\epsilon_{\max }=\frac{4 \epsilon_{0} \gamma_{\mathrm{e}}^{2}}{1+4 \epsilon_{0} \gamma_{\mathrm{e}}}$.

In the second step, both the radiation and the matter interact throughout the cross section of the IC process (Blumenthal \& Gould 1970):

$\sigma\left(x, \epsilon_{0}, \gamma_{\mathrm{e}}\right)=\frac{3 \sigma_{\mathrm{T}}}{4 \epsilon_{0} \gamma_{\mathrm{e}}^{2}} f(x)$

where $\sigma_{\mathrm{T}}$ is the Thomson cross section constant and $f(x)$ is:

$$
\begin{aligned}
f(x)= & {\left[2 x \ln x+x+1-2 x^{2}+\frac{\left(4 \epsilon_{0} \gamma_{\mathrm{e}} x\right)^{2}}{2\left(1+4 \epsilon_{0} \gamma_{\mathrm{e}} x\right)}\right] } \\
& \times P\left(1 / 4 \gamma_{\mathrm{e}}^{2}, 1, x\right)
\end{aligned}
$$

in which $x$ is:

$$
x=\frac{\epsilon}{4 \epsilon_{0} \gamma_{\mathrm{e}}^{2}\left(1-\epsilon / \gamma_{\mathrm{e}}\right)}
$$

and

$P\left(1 / 4 \gamma_{\mathrm{e}}^{2}, 1, x\right)=1$, for $1 / 4 \gamma_{\mathrm{e}}^{2} \leq x \leq 1$, and 0 otherwise. (11)

$P\left(1 / 4 \gamma_{\mathrm{e}}^{2}, 1, x\right)$ restricts the cross section to a physical case, where $\epsilon$ cannot be lower than $\epsilon_{0}$ or higher than $\epsilon_{\max }$.

The resulting IC spectral energy distribution in the observer's reference frame is:

$$
\begin{aligned}
\epsilon L_{\epsilon}= & \delta^{2+p} \epsilon^{2} \sum_{z_{\min }}^{z_{\max }} V_{\text {slice }}(z) \int_{\epsilon_{0}^{\min }(z)}^{\epsilon_{0}^{\max }(z)} \int_{\gamma_{\mathrm{e}}^{\min }(z)}^{\delta \gamma_{\mathrm{e}}^{\max }(z)} U_{\text {total }}\left(\epsilon_{0}, z\right) \\
& \times N\left(\gamma_{\mathrm{e}}, z\right) \sigma\left(x, \epsilon_{0}, \gamma_{\mathrm{e}}\right) \mathrm{d} \gamma \mathrm{d} \epsilon_{0} .
\end{aligned}
$$

Here, $\delta$ is the jet Doppler factor, viz. $\delta=\left[\Gamma_{\text {jet }}(1-\beta \cos \theta)\right]^{-1}$ and $\beta=v_{\text {jet }} / c$. $V_{\text {slice }}$ is a slice volume, which varies depending on the accuracy needed by each slice, $\theta$ is the angle between the jet and the observer line of sight, and $U_{\text {total }}\left(\epsilon_{0}, z\right)=$ $U_{\text {syn }}\left(\epsilon_{0}, z\right)+U_{\text {star }}\left(\epsilon_{0}, z\right)$, which is the total radiation energy density per seed photon energy unit and slice, taking into account both the synchrotron and the star radiation field components. The integral has been calculated numerically taking suitable integration steps from the behavior of the involved functions. We note that this is the optically thin case because, in the $\gamma$-jet conditions and at the EGRET energy range, the IC absorption coefficient is negligible. It is worth mentioning that the electron energy evolves following Eq. (2). Therefore, in order to obtain the desired high-energy photons, the frequency range for the seed photons will change with the evolution of the electron energies, since there is a link (the limits of Eq. (10) induced by the function $\left.P\left(1 / 4 \gamma_{\mathrm{e}}^{2}, 1, x\right)\right)$ between the incoming photon energy, the outgoing photon energy and the scattering electron energy. The electron Lorentz factor integration limits in Eq. (12) have been adopted following Georganopoulos et al. (2001).

\section{Application of the model}

\subsection{Parameter choice for LS 5039}

Although this model can be applied to other cases, we will test its prediction capabilities with LS 5039. The optical counterpart of LS 5039 is a bright $(V \sim 11)$ star of ON6.5 V((f)) spectral type (McSwain et al. 2004), at an estimated distance of $2.9 \mathrm{kpc}$ (Ribó et al. 2002). The bolometric luminosity of the companion star has been taken to be $L_{\mathrm{star}} \sim 1.2 \times 10^{39} \mathrm{erg} \mathrm{s}^{-1}$. A new value of the orbital period, $P=4.4267 \pm 0.0005$ days, the eccentricity, $e=0.48 \pm 0.06$, and the semi-major axis of the orbit, $a=2.6 \times 10^{12} \mathrm{~cm}\left(R_{\text {orb }}=a\right.$ except where otherwise stated), have been determined recently by McSwain et al. (2004). We adopt these values in our study. At radio wavelengths $(1.5-15 \mathrm{GHz})$, the spectral index was determined to be $\alpha \sim-0.5$ (where the flux density is $F_{v} \propto v^{\alpha}$ ) by Martí et al. (1998). From this spectral index, we have deduced the electron power law index $(p)$ following the known relationship $p=1-2 \alpha=2$. From VLBI observations (Paredes et al. $2000,2002)$, the radio constraint $v_{\text {jet }} \cos \theta \sim 0.15 c$ was found. The observed spectrum over $100 \mathrm{MeV}$ has been obtained from the third EGRET catalogue (Hartman et al. 1999). The total luminosity in the range $100-1500 \mathrm{MeV}$ is $2 \times 10^{35} \mathrm{erg} \mathrm{s}^{-1}$. The spectral energy index, $\eta=0.2 \pm 0.2$, corresponds to an observed photon index of $2.2 \pm 0.2$ (photon energy distribution $\phi_{\epsilon} \propto \epsilon^{-2.2}$ ). All these parameters are summarized in Table 1.

McSwain \& Gies (2002) proposed a value of $\theta=30^{\circ}$. Having this, and the radio constraint shown above $(\beta \cos \theta \sim$ $0.15)$, we obtain a $v_{\text {jet }} / c=0.2\left(\Gamma_{\text {jet }}=1.02\right)$. We stress that our model is weakly sensitive to changes on $v_{\text {jet }}$ and $\theta$ around the quoted values because of the adopted low value of the $\Gamma_{\text {jet }}$. Such a low $\Gamma_{\text {jet }}$ is not in disagreement with the value found in similar systems (e.g. 1.04 for SS 433, Spencer 1979; Hjellming $\&$ Johnston 1981). For LS 5039, we have assumed $\varpi=90^{\circ}$. In order to fix the $\gamma$-jet radius, we have imposed that $R_{\gamma}$ should 
Table 1. Known parameters for LS 5039.

\begin{tabular}{lcc}
\hline \hline Parameter & Description & Value \\
\hline$a$ & orbital semi-major axis & $2.6 \times 10^{12} \mathrm{~cm}$ \\
$L_{\text {star }}$ & star total luminosity & $1.2 \times 10^{39} \mathrm{erg} \mathrm{s}^{-1}$ \\
$\beta \cos \theta$ & relationship between the jet velocity and the jet-observer line of sight angle & 0.15 \\
$L_{\text {EGRET }}$ & luminosity at the EGRET band & $2 \times 10^{35} \mathrm{erg} \mathrm{s}^{-1}$ \\
$\eta$ & spectral energy index at the band $100-1000 \mathrm{MeV}$ & 0.2 \\
$\alpha$ & spectral index at the band $1.5-15 \mathrm{GHz}$ & -0.5 \\
\hline
\end{tabular}

Table 2. Fixed parameters used in our model for LS 5039.

\begin{tabular}{lcc}
\hline \hline Parameter & Description & Adopted value \\
\hline$\varpi$ & angle between the jet and the orbital plane & $90^{\circ}$ \\
$\Gamma_{\text {jet }}$ & jet Lorentz factor & 1.02 \\
$\theta$ & angle between the jet and the observer line of sight & $30^{\circ}$ \\
$R_{\gamma}$ & $\gamma$-jet radius & $10^{7} \mathrm{~cm}$ \\
$L_{\mathrm{ac}}$ & accretion disk luminosity & $10^{-8} M_{\odot} c^{2} \mathrm{yr}^{-1}$ \\
\hline
\end{tabular}

be neither wider than the inner disk (a few $10^{7} \mathrm{~cm}$ ) nor thinner than some few electron Larmor radii $\left(\sim 10^{6} \mathrm{~cm}\right)$. Thus, $R_{\gamma}$ has been taken to be $10^{7} \mathrm{~cm}$. Regarding $z_{0}$, since in this scenario $\left(R_{\text {orb }} \gg z_{0}\right)$ its size does not affect the results, it is taken to be zero. To determine the power of the jet, we need to fix also the accretion luminosity of the disk (Falcke \& Biermann 1996). A typical value for a microquasar with an O-type stellar companion can be $L_{\mathrm{ac}} \simeq 10^{-8} M_{\odot} c^{2} \mathrm{yr}^{-1}$. All these parameters are summarized in Table 2.

To determine $L_{\mathrm{ke}}$, we will use the jet-disk coupling hypothesis for Galactic jet sources of Falcke \& Biermann (1996): $L_{\mathrm{k}} \sim 0.1-0.001 L_{\mathrm{ac}}$, where $L_{\mathrm{k}}$ is the total kinetic luminosity of the jet. Since not all the jet power is carried just by the electron population, the former scaling relationship turns to $L_{\mathrm{ke}} \propto L_{\mathrm{ac}}$, where the prior scaling factor limits are now upper limits. In fact, $L_{\mathrm{ke}}$ will be fixed through comparison between the observed fluxes and the model. From the EGRET energy range, and the involved photon and electron energy relationships shown above, the initial maximum Lorentz factor of the electrons should be about $10^{4}$. A more accurate value will be found when we try to reproduce the observed spectrum slope. Regarding $B_{\gamma}$, we will study our model behavior along a wide range of magnetic values, from $B_{\gamma}$ close to estimated radio jet upper values to $100 \mathrm{G}$.

\subsection{Results}

First, we explore the range of validity of the free parameters of the model taking into account observational constraints. Once this is done, we can reproduce the observational data with our model. Finally, additional comments on the model are made.

\subsubsection{Exploring $B_{\gamma}$ and $\gamma_{\mathrm{e} 0}^{\max }$}

To determine the behavior of the model with magnetic field strength, we have run our model for different values of the magnetic field strength and a fixed value of $\gamma_{\mathrm{e} 0}^{\max }=5 \times 10^{4}$. We show at the top of Fig. 1 the computed IC spectral energy distribution $\left(\epsilon L_{\epsilon}\right)$ for three representative cases $(1,10$ and $100 \mathrm{G})$. The spectral slopes in the energy range $100-1000 \mathrm{MeV}$, where EGRET detected the source, are similar. Since our model generates similar spectra for a wide range of magnetic field values, we have to focus our attention on the distances reached by the $\gamma$-jet, which is strongly dependent on $B_{\gamma}$. Then, high magnetic fields (tens of G or higher) would imply a $\gamma$-jet evolving very fast, whereas low magnetic fields (few $\mathrm{G}$ or lower) would imply a $\gamma$-jet evolving smoothly (see Sect. 4.2.3, Figs. 3 and 4). Moreover, regarding values well below $1 \mathrm{G}$, we notice that upper limits of $0.2 \mathrm{G}$ have been estimated from radio observations (Paredes et al. 2002) for the magnetic field in the radio jet. Therefore, $B_{\gamma}$ of about several tenths of $\mathrm{G}$ could be taken as a lower limit.

In order to explore the dependence of the IC spectral energy distribution on $\gamma_{\mathrm{e} 0}^{\max }$, three $\gamma_{\mathrm{e} 0}^{\max }\left(10^{4}, 5 \times 10^{4}\right.$ and $\left.10^{5}\right)$ have been used, fixing $B_{\gamma}=10 \mathrm{G}$. The results are shown at the bottom of Fig. 1. For $\gamma_{\mathrm{e} 0}^{\max }=10^{4}$, the computed $\eta$, about 0.7 , is $2.5 \sigma$ softer than that given by EGRET observations. Therefore, $\gamma_{\mathrm{e} 0}^{\max }>10^{4}$ is needed. An upper limit cannot be clearly fixed yet, since we do not know properly the spectral energy cutoff.

\subsubsection{Reproducing the EGRET data}

The computed IC spectral energy distributions for two different values of the magnetic field are shown in Fig. 2. We also show the EGRET data points of 3EG J1824-1514 (Hartman et al. 1999). The kinetic luminosity has been adopted such that the level of the observed flux at $100 \mathrm{MeV}$ can be reproduced. The parameter values used in both cases are summarized in Table 3. Since we are dealing with an eccentric system, the star's radiation density in the jet is affected by the orbital distance variation. We have explored the importance of this effect by computing the IC spectral energy distribution at 


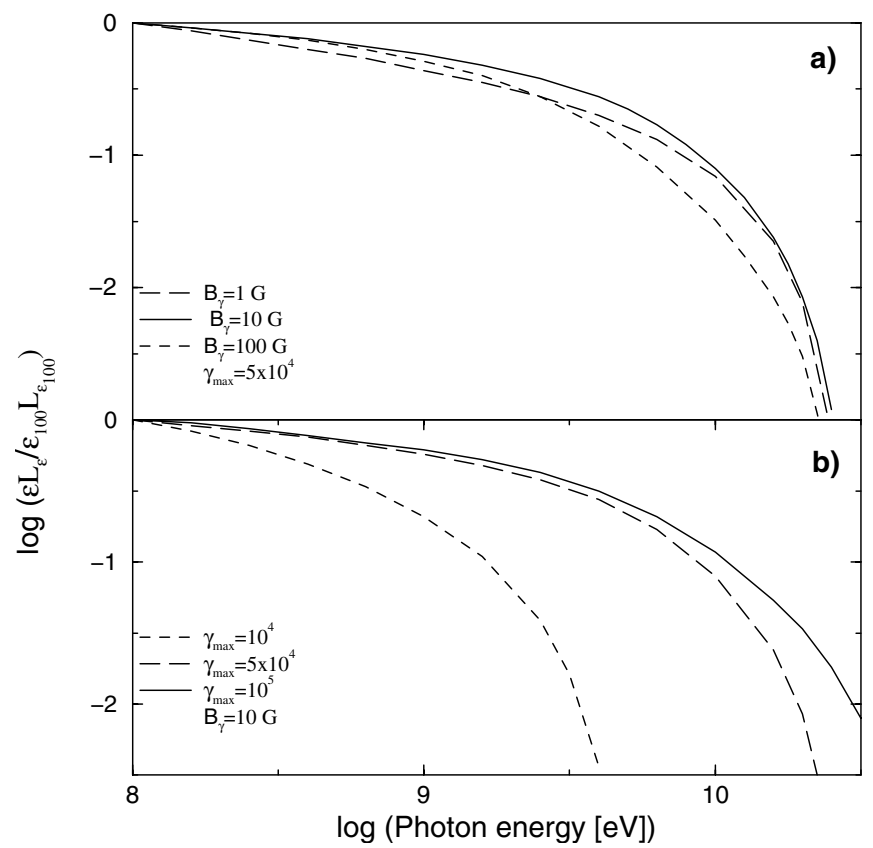

Fig. 1. Computed IC spectral energy distributions normalized to their value at $100 \mathrm{MeV}$ for different values of the magnetic field strength and the maximum electron Lorentz factor. a) For values of the magnetic field of 1,10 and $100 \mathrm{G}$ and fixing the maximum Lorentz factor at $5 \times 10^{4}$. b) For values of the maximum Lorentz factor of the electrons of $1 \times 10^{4}, 5 \times 10^{4}$ and $1 \times 10^{5}$ and fixing the magnetic field to $10 \mathrm{G}$.

Table 3. Parameter values used in the model for Fig. 2.

\begin{tabular}{lcc}
\hline \hline Parameter & top & bottom \\
\hline$B_{\gamma}$ & $1 \mathrm{G}$ & $10 \mathrm{G}$ \\
$L_{\mathrm{ke}}$ & $10^{36} \mathrm{erg} / \mathrm{s}$ & $3 \times 10^{36} \mathrm{erg} / \mathrm{s}$ \\
$\gamma_{\mathrm{e} 0}^{\max }$ & $10^{5}$ & $10^{5}$ \\
\hline
\end{tabular}

periastron and apastron passage as well as for $R_{\mathrm{orb}}=a$. In the case $B_{\gamma}=10 \mathrm{G}$, since SSC is strongly dominant, the effect of the orbital eccentricity on the computed IC luminosity is not significant. However, for a $B_{\gamma}=1 \mathrm{G}$, the EC scattering is dominant and the orbital distance variation between apastron and periastron passages produces a change of about 50 per cent in the computed IC luminosity. At the top of Fig. 2, we have plotted our results obtained at three orbital distances: $1.35 \times 10^{12} \mathrm{~cm}$ for periastron, $2.6 \times 10^{12} \mathrm{~cm}$ for the semi-major axis of the orbit and $3.85 \times 10^{12} \mathrm{~cm}$ for apastron. Since the viewing periods of EGRET are longer than the orbital period of the system, we are not able to relate the variability predictions of our model to the variability detected by EGRET. As can be seen in Fig. 2, our model reproduces properly the EGRET data below about $1 \mathrm{GeV}$. Beyond this energy, the computed spectrum becomes significantly softer, but it is not in disagreement with the upper limits given by EGRET at this energy range.

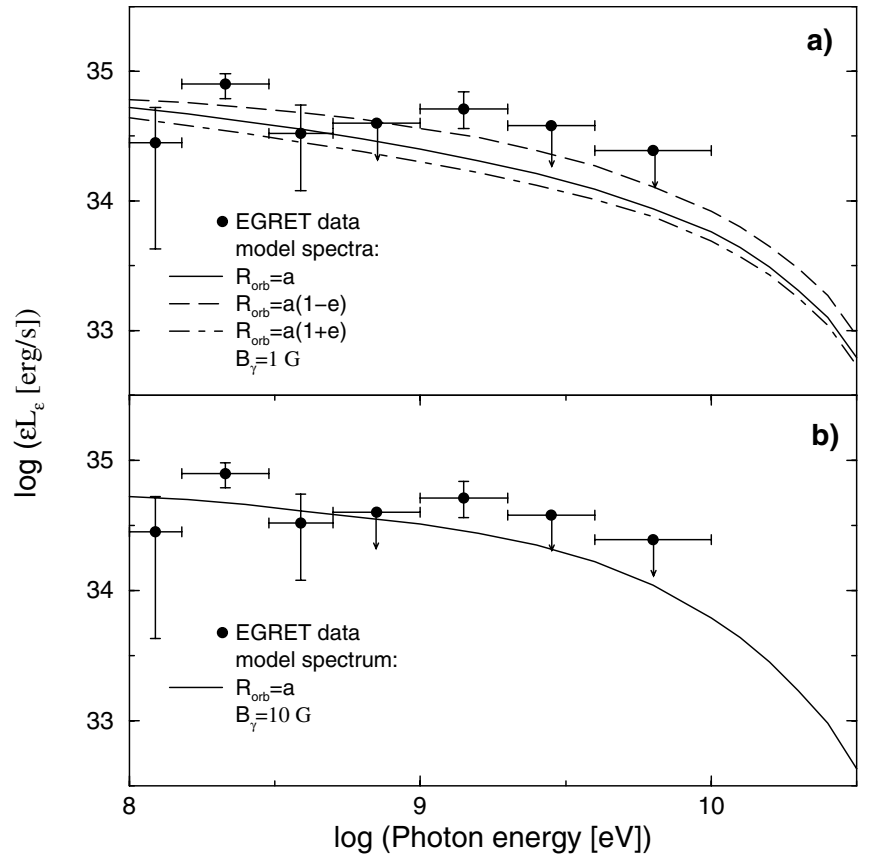

Fig. 2. Two IC spectral energy distributions computed with the present model using the physical parameters of Table 3. The EGRET data points are also shown. The upper limits on undetected EGRET points are plotted with arrows. a) A magnetic field of $1 \mathrm{G}$ has been assumed. Also, the IC spectral energy distribution for both the apastron and the periastron passage are shown. b) A magnetic field of $10 \mathrm{G}$ has been assumed.

\subsubsection{Further comments}

Additional comments have to be made about the magnetic field in the $\gamma$-jet. We have plotted the temporal evolution of the synchrotron and companion star radiation energy densities for both a $B_{\gamma}$ of $1 \mathrm{G}$ and a $B_{\gamma}$ of $10 \mathrm{G}$ within a given slice moving along the jet (Figs. 3 and 4 ). It shows that a variation of one order of magnitude in $B_{\gamma}$ implies a variation of more than two orders of magnitude in the synchrotron radiation energy density. Therefore, in the context of our model, magnetic fields between 1 and $10 \mathrm{G}$ or higher could be tightly related to the high-energy $\gamma$-ray emission in LS 5039, as the SSC loss process is important and even dominant. Finally, we have explored the infrared synchrotron emission from the $\gamma$-jet. Taking a $B_{\gamma}=10 \mathrm{G}$, we have obtained a total luminosity in the range $10^{12}-10^{14} \mathrm{~Hz}$ of about $10^{32} \mathrm{erg} \mathrm{s}^{-1}$ for LS 5039. If we compare this value to the luminosity of the companion star in this frequency band, we find that the infrared jet luminosity is five orders of magnitude lower.

\section{Discussion}

As we have shown, our model is able to reproduce the highenergy $\gamma$-ray emission of the EGRET source 3EG J1824-1514 by assuming its origin in the microquasar LS 5039. Since there are no special constraints associated with a particular source in our model, it could be applied to other unidentified EGRET sources that might be also associated with microquasars. A maximum electron Lorentz factor higher than $10^{4}$ is 


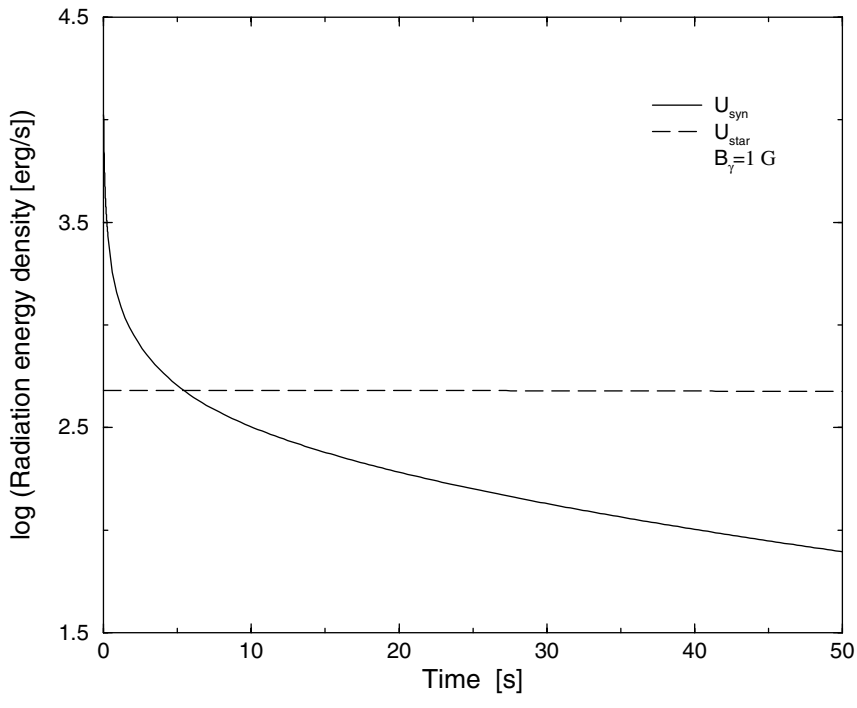

Fig. 3. The evolution of the synchrotron and companion star radiation energy densities within a given slice. The value of the magnetic field is $B_{\gamma}=1 \mathrm{G}$ and the maximum Lorentz factor of the electrons is $5 \times 10^{4}$. The time axis finishes when a given slice moving along the jet stops emitting due to losses through the IC effect at $100 \mathrm{MeV}$.

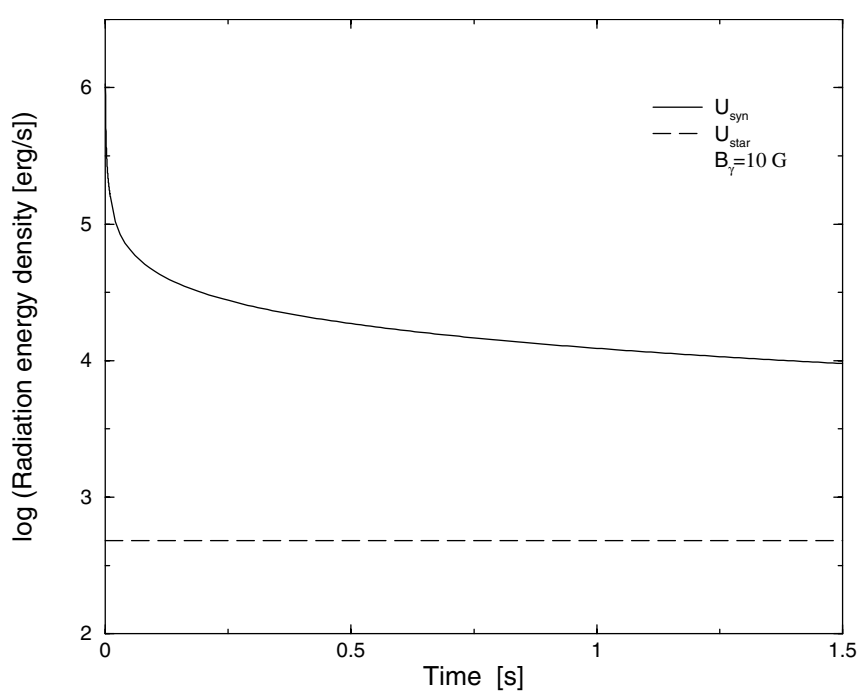

Fig. 4. Same as Fig. 3 but for $B_{\gamma}=10 \mathrm{G}$.

a good parameter range to obtain the observed spectral slope. An upper limit on $\gamma_{\mathrm{e} 0}^{\max }$ has not been determined yet due to the lack of knowledge about the high-energy cutoff. Further observations by the next, more sensitive, hard $\gamma$-ray instruments $\left(\mathrm{AGILE}^{2}, \mathrm{GLAST}^{3}\right.$ ) are needed to determine this cutoff. To reproduce the observed luminosities, $L_{\mathrm{ke}} \sim 10^{36} \mathrm{erg} / \mathrm{s}$ is needed for a magnetic field of $1 \mathrm{G}$, and $L_{\mathrm{ke}} \sim 3 \times 10^{36} \mathrm{erg} / \mathrm{s}$ is needed for a magnetic field of $10 \mathrm{G}$. Both values for $L_{\mathrm{ke}}$ are well within the range of allowed values established in the parameter discussion.

For LS 5039, regarding the intensity of the magnetic field, the SSC effect would be dominant for values of about $10 \mathrm{G}$ or higher, and at least non-negligible for values of about $1 \mathrm{G}$.

\footnotetext{
${ }^{2}$ http://agile.mi.iasf.cnr.it

${ }^{3}$ http://glast.gsfc.nasa.gov
}

In the second case, due to the importance of the star's seed photons, variations in the orbital distance have a significant effect on the IC flux on the scale of the orbital period of the system.

In the case of high $B_{\gamma}$ (above $10 \mathrm{G}$ ), due to the strong energy losses, the electrons might need to be reaccelerated after leaving the $\gamma$-jet to reach the observed radio jet. However, for $B_{\gamma}=1-10 \mathrm{G}$, reacceleration might not be necessary in regions closer to the compact object than the radio jet.

Our approach is an attempt to join radiation and matter in a complete physical way. The introduction of losses is a necessary step to obtain realistic results, and a way to determine at the end the importance that these losses can have. The importance of the synchrotron process gives strong relevance to the magnetic field. Such a question is relevant studying the seed photon origin in the IC interaction and the timescales of the energy processes involved, and it is also related to variability. Additional elements in our scenario such as the corona, disk, environment interaction, reacceleration processes, pair creation-annihilation phenomena and accretion variability will be considered in future studies. Also, emission at other wavelengths will require a proper scenario, different from the one presented here.

Although future observations have to be done to confirm the nature of LS 5039 as a $\gamma$-ray emitter, our numerical model is able to produce significant $\gamma$-ray emission levels. AGILE, an italian hard $\gamma$-ray satellite expected to be in orbit in 2005, with an angular resolution two times better than EGRET, will make it possible to improve the accuracy of the hard $\gamma$-ray source position. Also, its sensitivity, almost three times better than that of EGRET, could define more clearly the spectral shape and its cutoff. The next generation of hard $\gamma$-ray satellites represented by GLAST, which will be put in orbit around 2008, will improve even more the study of the variability and spectral features at this energy range, in order to better understand the physics underlying the very high energy processes.

Acknowledgements. We are grateful to Gustavo Romero, Marc Ribó, and Joan García-Sánchez for their useful comments and suggestions. V.B.-R. and J.M.P. acknowledge partial support by DGI of the Ministerio de Ciencia y Tecnología (Spain) under grant AYA-2001-3092, as well as additional support from the European Regional Development Fund (ERDF/FEDER). During this work, V.B.-R. has been supported by the DGI of the Ministerio de Ciencia y Tecnología (Spain) under the fellowship FP-2001-2699.

\section{References}

Atoyan, A. M., \& Aharonian, F. A. 1999, MNRAS, 302, 253

Band, D. L., \& Grindlay, J. E. 1986, ApJ, 311, 595

Blumenthal, G. R., \& Gould, R. J. 1970, RMP, 42, 237

Collmar, W. 2003, Proc. 4th AGILE Science Workshop, Frascati (Rome) on 11-13 June 2003

Dermer, C. D., Schlickeiser, R., \& Mastichiadis, A. 1992, A\&A, 256, L27

Falcke, H., \& Biermann, P. L. 1996, A\&A, 308, 321

Fender, R. 2004, Compact Stellar X-Ray Sources, ed. W. H. G. Lewin, \& M. van der Klis (Cambridge University Press), to appear 
Georganopoulos, M., Kirk, J. G., \& Mastichiadis, A. 2001, ApJ, 561, 111

Georganopoulos, M., Aharonian, F. A., \& Kirk, J. G. 2002, A\&A, 388, L25

Ghisellini, G., Maraschi, L., \& Treves, A. 1985, A\&A, 146, 204

Hartman, R. C., Bertsch, D. L., \& Bloom, S. D., et al. 1999, ApJS, 123,79

Hjellming, R. M., \& Johnston, K. J. 1981, ApJ, 246, L141

Kaufman Bernadó, M. M., Romero, G. E., \& Mirabel, I. F. 2002, A\&A, 385, L10

Markoff, S., Falcke, H., \& Fender, R. 2001, A\&A, 372, L25

Martí, J., Paredes, J. M., \& Ribó, M. 1998, A\&A, 338, L71

McSwain, M. V., \& Gies, D. R. 2002, ApJ, 568, L27

McSwain, M. V., Gies, D. R., Huang, W., et al. 2004, ApJ, 600, 927

Mirabel, I. F., \& Rodríguez, L. F. 1999, ARA\&A, 37, 409
Pacholczyk, A. G. 1970, Radio Astrophysics (San Francisco, CA: Freeman)

Paredes, J. M., Martí, J., Ribó, M., \& Massi, M. 2000, Science, 288, 2340

Paredes, J. M., Ribó, M., Ros, E., Martí, J., \& Massi, M. 2002, A\&A, 393, L99

Ribó, M., Paredes, J. M., \& Romero, G. E., et al. 2002, A\&A, 384, 954

Ribó, M. 2002, Ph.D. Thesis, Universitat de Barcelona

Romero, G. E., Kaufman Bernadó, M. M., \& Mirabel, I. F. 2002, A\&A, 393, L61

Romero, G. E., Torres, D. F., Kaufman Bernadó, M. M., \& Mirabel, I. F. 2003, A\&A, 410, L1

Spencer, R. E. 1979, Nature, 282, 483 